\title{
Un atelier d'écriture créative en FLE
}

Tissages et apprentissages plurilingues

\section{Chantal Dompmartin-Normand et Anne Le Groignec}

\section{(2) OpenEdition Journals}

Édition électronique

URL : http://journals.openedition.org/esp/539

DOI : $10.4000 /$ esp.539

ISSN : 2532-0319

Éditeur

Centre d'Information sur l'Éducation Bilingue et Plurilingue

Édition imprimée

Date de publication : 1 juin 2015

Pagination : 59-71

ISSN : 1127-266X

Référence électronique

Chantal Dompmartin-Normand et Anne Le Groignec, « Un atelier d'écriture créative en FLE », Éducation et sociétés plurilingues [En ligne], 38 | 2015, mis en ligne le 01 mars 2016, consulté le 25 février 2021. URL : http://journals.openedition.org/esp/539; DOI : https://doi.org/10.4000/esp.539 


\section{UN ATELIER D'ÉCRITURE CRÉATIVE EN FLE: TISSAGES ET APPRENTISSAGES PLURILINGUES}

\section{Chantal DompMartin-Normand, Anne Le GroigneG}

Ci troviamo sul campo di un laboratorio di scrittura creativa, all'interno di un cursus di francese lingua straniera e L2 (FLE/FLS), all'università. Gli studenti-partecipanti sono in fase di acquisizione-apprendimento di una lingua aggiuntiva al loro repertorio, il francese, con il suo orizzonte linguistico normativo e le rappresentazioni associate. Il lavoro proposto nel laboratorio tende a cambiare la rappresentazione di quest'orizzonte, spostando il desiderio legittimo di "migliorare il proprio francese" verso una dimensione creativa in grado di aprire nuove porte. La lingua target dell'apprendimento potrebbe cosi diventare l'occasione di mettersi in gioco, in risonanza con le altre lingue già acquisite dai soggetti scriventi. La nuova lingua poterbbe cosi non essere più solo un luogo di sforzi dovuti a "mancanza di parole", ma trasformarsi in una lingua dei possibili per dire quello che si ha da dire. La ricerca ha l'obiettivo di rivelare ed analizzare le tracce e gli indizi di questi spostamenti di rappresentazioni, pratiche e relazioni di scrittura.

Parole chiavi: Laboratorio di scrittura creativa, FLE/FLS, lingua aggiuntiva, lingua dei possibili, rappresentazioni, pratiche

We are in a creative writing workshop with foreign students learning French at the university. The students are clearly on their way to "a better French", trying to improve it and rarely forgetting the norms and images of ideal progress. The workshop aims to activate their representations and orientations within the creative writing process. Thus, the target language can interact and develop a resonance with the languages previously present in their repertoire. The additional language can cease to be only a restricted means of expression due to lack of words, and become a language of possibly meaningful narratives. In this research, we try to collect and analyze the hints and signs of the transformations potentially triggered by the workshop with regard to students' representations, practices and how they relate to writing.

Key words: Creative writing workshop, French as a Foreign or Second Language, additional language, language of the possibles, representations, practices

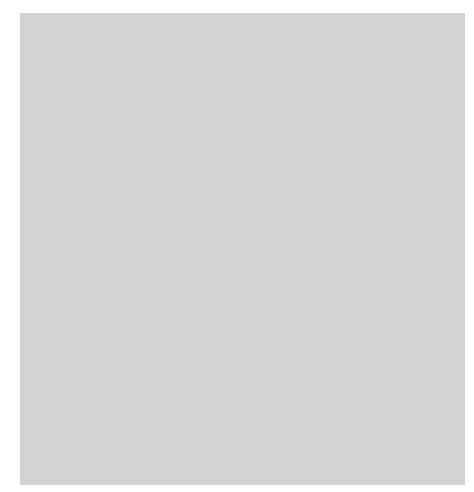

«C'est plus facile d'apprendre une langue quand on a quelque chose à dire» (Natalia-Pologne-2013-ALG-voir note 5)

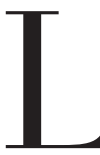

a recherche-intervention dont il sera question ici a pour cadre un atelier d'écriture créative à l'université de Toulouse au Département d'Études de Français Langue Etrangère. Cet atelier est à visée de formation, en l'occurrence linguistique. Il est proposé, en tant que module optionnel dans un parcours d'apprentissage de français langue étrangère et seconde, à des étudiants étran- 
Un atelier d'écriture créative en FLE: tissages et apprentissages plurilingues

C. DOMPMARTIN-NORMAND,

A. Le Groignec gers, nommés aussi «allophones». Si le premier terme renvoie à l'ailleurs géographique et culturel d'où ils viennent, le second renvoie à leur altérité linguistique. Ils parlent et écrivent d'«autres langues», que celle qui constitue leur projet d'apprentissage actuel, à savoir leurs(s) langue $(\mathrm{s})$ premières(s) et celles acquises par la suite dans leur itinéraire scolaire et social. Ils ont déjà un niveau «avancé» en français (B2-C1), l'ayant acquis dans ce cadre universitaire ou dans des cadres antérieurs, scolaires et non scolaires. Certains d'entre eux vivent en France, i.e. dans un lieu où la langue est fortement socialisée en dehors du cadre des cours, depuis plusieurs années; d'autres sont ici depuis peu. Pour certains, la langue était circulante dans leur pays à plus ou moins large échelle (par exemple Maghreb ou pays africains francophones); pour d'autres elle était à un moment donné vraiment nouvelle, vraiment étrangère. La langue française est ainsi additionnelle au répertoire varié des sujets, occupant pour chacun une place évolutive sur un continuum, qui va de l'altérité maximale à un sentiment d'incorporation.

Plutôt que seulement allophones - rappelons que ce terme a été inventé pour remplacer la dénomination négative «non-francophone» - car le français est de facto un déjà-là dans leur vie, les apprenants sont avantageusement dénommés «culturellement et linguistiquement divers» dans les courants de recherche nord-américains (Prasad 2013) ou «plurilingues» dans le courant de recherche européen de la didactique du plurilinguisme (Billiez 1998). Dans ce courant émerge, à partir des années 1990, la notion de compétence plurilingue et pluriculturelle, développée en particulier dans les travaux du Conseil de l'Europe. Cette notion valide l'hétérogénéité des ressources et tente de proposer un cadre conceptuel où les langues seraient réellement en contact dans le répertoire d'un sujet et ne progresseraient pas séparément les unes des autres (1).

Dans ce paradigme, toute langue nouvelle peut être vue comme simple ressource supplémentaire dans une boite à outils langagière, pragmatiquement mise en œuvre en fonction des nécessités comme nous l'avons montré dans une étude portant sur le trilinguisme familial (Dompmartin et alii. 2013). Or cette boite, si elle n'est plus tout à fait «boite noire», beaucoup de processus cognitifs, sociolinguistiques et psychoaffectifs étant décrits, n'en reste pas moins encore à explorer, surtout du fait que les statuts des outils qu'elle contient évoluent en permanence pour le locuteur. Ainsi, dans la mesure où elle est visée par l'apprentissage à un moment donné dans le parcours d'un individu - et de ce fait nommée «langue-cible», une langue jouit et pâtit provisoirement d'un statut particulier, chargé d'intentionnalité et d'affects, sensible aux variations de motivation et tributaire de la qua- 
Un atelier d'écriture créative en FLE: tissages et apprentissages plurilingues

C. DOMPMARTIN-NORMAND,

A. Le Groignec lité d'un rapport que le sujet entretient avec elle. C'est sur ce plan-là, du rapport à la langue et des représentations la concernant, que se situent notre intervention didactique et notre recherche.

Nous postulons d'abord que le goût de la langue n'est pas un donné, ni le plaisir de celle-ci entretenu suffisamment dans le $(\mathrm{s})$ processus d'apprentissage, d'autant que ces aspects sont souvent occultés ou écrasés par le discours de la performance qui recouvre le champ éducatif et social actuellement. Ainsi, la difficulté (de la langue) est fréquemment abondamment décrite par les usagers (étrangers ou autochtones), le plaisir des mots, du déplacement identitaire et de l'exploration de l'altérité étant par comparaison peu thématisé. Il est certain qu'à l'université, dans les cursus d'enseignement-apprentissage des langues d'une manière générale, les genres académiques issus de la tradition continuent de tenir le haut du pavé dans les représentations mentales de quasiment tous les acteurs. C'est à l'aune de ces productions académiques, qui privilégient le discours critique et argumentatif, que s'évalue en grande partie la compétence en langue, et ce surtout à l'écrit et de façon croissante au fur et à mesure des niveaux de langue visés.

En contrepoint à cette tendance, se développent les démarches artistiques où la créativité est remise à l'honneur et l'atelier d'écriture que nous animons cherche sa place précisément dans cette orientation. Nous cherchons dans le cheminement à susciter un travail sur les représentations (qui, selon nous, aurait une incidence sur la cognition). Notre recherche de type ethnographique, qualitatif et réflexif a pour objectif de repérer les traces de ce qui se passerait en plus de l'acquisition d'une langue donnée: la progression d'habiletés transverses, le déplacement de représentations sur la difficulté de la langue, le rapport à l'écriture en langue «autre» et en général, dans sa dimension productive de mise en mots de soi et de son expérience.

Comme annoncé, l'objectif de l'atelier, tel qui est perçu par les étudiants, légitimé par le contrat didactique, présent dans les représentations des autres acteurs du contexte (hiérarchie, collègues...) porte sur le français. Interrogés sur leurs attentes en début d'atelier, les étudiants déclarent: «je veux améliorer mon français, améliorer ma grammaire, augmenter mon vocabulaire...». Tout est focalisé sur le désir d'augmentation/amélioration de compétence dans la nouvelle langue. Ce qui semble parfaitement normal et légitime dans le cadre, surtout si on est conscient que le champ de représentations reste balisé par l'image du locuteur natif comme locuteur parfait «possédant» la «maitrise» de la langue. A contrario le locuteur étranger ne possèderait rien, et la langue serait pour lui indomptée. La langue apparaît donc comme un objet réifié, et les connaissances et habiletés linguis- 
Un atelier d'écriture créative en FLE: tissages et apprentissages plurilingues

C. DOMPMARTIN-NORMAND,

A. Le Groignec
PAROLES D'ÉCRIVAINS tiques comme séparées entre langue «maternelle» et langue(s) «étrangère(s)». En effet, bien que l'existence des processus inter- ou translinguistiques soit perçue par les acteurs, enseignants et apprenants de langue(s), elle l'est de façon restreinte. La notion d'interférence, identifiée depuis longtemps, est plutôt interprétée en termes d'obstacle, d'entrave, de faux contact, de problème, de «transfert négatif» (Hamers et Blanc 1983) (2). Celle de «transfert positif» (Cummins 2013), qui signifie la prise d'appui entre les langues dans le répertoire d'un sujet, peine semble-t-il à intégrer les discours et représentations, malgré les travaux de recherche en didactique du plurilinguisme mentionnées plus haut. Ceux-ci, ancrés disciplinairement dans la sociolinguistique, visent à valoriser ce pôle positif des phénomènes depuis quelques décennies déjà. Ont ainsi émergé des courants et des discours scientifiques qui autorisent à penser le translinguistique et les continuités. «La réflexion et le discours politique et scolaire récents montrent que le français s'est peu à peu fait à l'idée qu'il lui fallait vivre avec d'autres langues, non pas dans une co-existence indifférente ou dans une peur quasiment maladive de la contagion de l'autre, mais en sachant, comme le dit si bien Glissant (1993) que "ce n'est pas une question de parler des langues, ce n'est pas le problème. On peut ne pas parler d'autres langues que la sienne. C'est plutôt la manière même de parler sa propre langue, de la parler de manière fermée ou ouverte; de la parler dans l'ignorance de la présence des autres langues ou dans la prescience que les autres langues existent et qu'elles nous influencent même sans qu'on le sache"» (Perregaux 2004: 147).

Sur le terrain de l'apprentissage, nous cherchons d'une manière générale les démarches didactiques et dispositifs qui permettraient de traduire dans le concret de la classe d'une langue le cadre théorique précité. Et l'atelier semble bien un lieu privilégié, pour toucher par le détour une dimension autre, en ce qu'il permettrait de déplacer le focus. Dans l'atelier, contrairement aux autres cours de sa semaine, l'étudiant établirait un autre rapport à la langue et à l'apprentissage, en oubliant le caractère étranger de cette langue. L'idée, c'est qu'à partir du manque - de mots, de matériau linguistique - il puisse y avoir une élaboration dans les représentations de gains, de plus. Les plus ne sont pas perceptibles immédiatement. Doit être dite peut-être d'abord la souffrance de l'étrangéité, de l'exil et du déplacement. Le chemin demande élaboration au sens psychique du terme, ce que laissent entendre des écrivains plurilingues ayant choisi une langue autre que première comme moyen d'expression, au moins à une étape de leur existence.

Akira Mizubayashi écrit: «Je suis locataire de la langue française». Il raconte comment il a plongé dans cette langue en raison de son ressenti de manque de mots dans sa langue première. Il raconte com- 
Un atelier d'écriture créative en FLE: tissages et apprentissages plurilingues

C. DOMPMARTIN-NORMAND,

A. Le Groignec ment écrire en français lui a permis autre chose, a ouvert un autre champ pour lui. Il va jusqu'à déclarer la langue française comme sa langue paternelle, son père étant par ailleurs la figure encourageante de son éducation, de son passage à l'âge adulte, de son émancipation. Il dit aussi la dualité, la double étrangéité, qu'il revendique, tout en se déclarant «faux bilingue».

«Je suis étranger ici et là et je le demeure. [...] Je revendique sans honte ni tristesse mon étrangéité: ce double statut d'étranger que je porte en moi, qui me permet de tendre sans cesse vers une perspective sur le réel qui est celle de l'Autre. [...] Avec la meilleure chance du monde, je ferais partie des faux bilingues» (Mizubayashi 2011: 268).

Il se réfère à Nancy Huston qui écrit aussi qu'elle est une «fausse bilingue» (Huston 1999: 53). Elle décrit les ambiguités, les frottements et les manques dus à la «double absence» (Sayad 1999). Elle évoque le masque et la mise en scène de soi qui est rendue possible dans la langue autre. Et elle parle des possibles ouverts dans cette langue-là.

«Le je que j’utilisais si librement dans mes écrits était aussi, pas de doute, l'un des effets de ma connaissance déracinée. Une certaine absence de honte était rendue possible par le fait que j'écrivais dans une langue étrangère - en partie parce que, au moins dans mon imagination, mes parents ne parlaient pas cette langue, mais plus encore parce que pour moi le français n'avait rien à voir avec ma vie intime, intérieure. En français je pouvais dire, très calmement et avec une certaine indifférence, des choses qu'il m'aurait été difficile de révéler ou même de penser dans ma langue maternelle» (notre traduction) (Huston 2005) (3).

Julia Kristeva dans Etrangers à nous-même décrit le caractère irréductible de l'étrangéité et de l'exil; elle mentionne la dureté de la coupure entre les langues (et derrière elles les identités) avec néanmoins la possibilité d'un «[...] miracle [...] qui soude l'origine à l'acquis dans une de ces synthèses mobiles et novatrices dont sont capables les grands savants et les grands artistes immigrés» (Kristeva 1988: 49). Elle semble toutefois réserver le miracle à une minorité de locuteurs qualifiés de «grands». Notre hypothèse est que justement tout locuteur plurilingue peut accéder à des «soudures», grâce à la pratique artistique, en l'occurrence l'écriture. Et au terme soudure nous préférons tissage, à l'instar de nos collègues nord-américains qui conceptualisent le «code meshing» (Canagarajah 2011).

Nous formulons ainsi l'hypothèse que le développement de la littératie dans une langue donnée participe au développement d'une littératie holistique, de façon intégrative et subtile, surtout si et peut-être seulement si l'entre-langues est mis en travail dans une pratique artistique. 
Un atelier d'écriture créative en FLE: tissages et apprentissages plurilingues

C. DOMPMARTIN-NORMAND,

A. Le Groignec

\section{TRAVAILLER} L'ENTRE-LANGUES DANS L'ATELIER
L'atelier réunit un groupe d'une quinzaine de participants et prévoit un temps hebdomadaire de 4 heures d'affilée pendant 12 semaines. Il s'organise autour d'invariants tels que ceux décrits par Claire Boniface (1992: 13), avec des aménagements locaux. En premier arrive une proposition d'écriture, écrite par l'animateur. Elle comprend la lecture et l'oralisation d'un texte d'auteur avec éventuellement un document où cette oralisation est prise en charge par l'auteur lui-même (Grand Corps Malade en vidéo: Rencontres), ou un comédien (Samy Frey sur la scène d'un théâtre parisien, récitant des extraits de Fe me souviens de Georges Perec). Sont utilisés aussi d'autres types de documents: tableaux, photographies... Après ce temps déclencheur /1/ et l'imprégnation/travail de compréhension des textes ou œuvres et de leurs cotextes, vient un temps d'écriture individuelle /2/. Quelquefois la consigne de départ est celle d'un «écrire à la manière de...». L'amorce est alors celle de l'écriture d'un autre. D'autres fois, la consigne est élaborée à partir d'autres contraintes et libertés. Puis intervient un temps de socialisation des textes dans leurs premiers jets /3/. Les échanges qui le constituent obéissent à des règles définies dans une charte: il s'agit de s'exprimer sur le texte, de dire ce qu'il nous évoque, en quoi il nous intéresse, nous intrigue, nous touche. Qu'avons-nous compris ou pas encore bien compris? Où faut-il ajouter des éléments de contexte?

Commence alors la phase de réécriture /4/, comprenant une phase de correction morphosyntaxique (parole de l'enseignant), visant à l'autocorrection ou à la reformulation prise en charge par l'apprenant, toujours dans le but d'être mieux compris (prise en compte du feedback du groupe). L'amélioration de l'expression en français est subordonnée à cette dimension de la réception du texte par ces premiers auditeurs. Il s'agit de donner aux auteurs confiance dans leur capacité d'écrire, qu'ils puissent s'autoriser à différer la stricte correction morphosyntaxique de leur texte, en leur faisant toucher du doigt que ce n'est pas la condition préalable à l'expression. En fin de processus, il est prévu que chaque étudiant constitue un recueil de ses textes du semestre, avec le travail de mise en page et la réalisation d'un objet final, dont on souligne la dimension potentielle de «bel objet». De surcroit, dans notre cas, est proposée une publication sur un blog de morceaux choisis. Rien n'est obligatoire (ni même l'oralisation des textes en phase /2/ si la personne n'est pas prête). Le principe énoncé est celui de l'appartenance du texte à son auteur.

Les ateliers se déroulent dans ce cadre avec ces repères, simples balises pour un cheminement collectif, susceptibles d'être elles aussi déplacées. En particulier, la consigne peut être modifiée, 
Un atelier d'écriture créative en FLE: tissages et apprentissages plurilingues

C. DOMPMARTIN-NORMAND,

A. Le Groignec

\section{L'AILLEURS \\ ET LICI, LE PASSÉ ET PRÉSENT, LA PROFONDEUR ET L'AFFLEUREMENT DES MOTS}

réorientée à la lumière des échanges de la phase /3/, ce qui situe la démarche dans un cadre de co-construction créative, relevant de l'art participatif. L'animatrice-enseignante pré-teste la proposition et dans certains cas poursuit son écriture en même temps que les étudiants, mettant par là à l'épreuve la pertinence des consignes et se mettant en situation (et en danger) de partager également son texte, ses doutes d'auteur quant à son intérêt, ses émotions éventuellement perturbantes, son étonnement devant ce qui est arrivé sur le papier.

Dans ce qui suit, nous essayons de faire partager à nos lecteurs quelques-uns des fils de ce qui s'est produit dans les ateliers et qui laisse entrevoir, dans les textes et dans l'expérience d'écriture commentée, les passages de l'ailleurs à l'ici, de l'avant au temps présent, et la profondeur de soi que les participants donnent à entendre et à lire. La construction dans le processus d'habiletés langagières transverses apparait. Nous observerons des figures de style mises en œuvre dans ce travail d'écriture avant de dégager des «plus» inattendus auxquels accèdent les participants-écrivains.

L'écrivain s'efforce d'écrire «ici» quelque chose de «loin».

«Quand j'écris, je suis loin» dit Naoko (Japon-2013-ALG) (4). Cette réflexion résume un ensemble de phénomènes convergents que nous relevons dans les ateliers. «Je» pourrait donc être présent(e) à la fois ici, en train d'écrire, et loin. Dans cette ubiquité, nous entendons aussi les questions: «où suis-je quand j'écris?» et «d'où est-ce que j'écris?». Une des motivations des étudiants plurilingues en atelier semble donc d'apporter et de faire entendre dans un «ici» quelque chose de «loin». Quelque chose d'audible, de compréhensible, qui fasse sens pour soi et pour les autres, auditeurs et lecteurs.

Ici représente certes la France et le cadre institutionnel, académique qui les évalue et valide ou non leur «niveau» de langue, mais c'est aussi le groupe plurilingue des participants à l'atelier d'écriture, à la fois écrivant eux-mêmes, lecteurs et auditeurs. C'est aussi l'espace de la page, blanche au départ et bien concrètement présente comme un ici où produire et exister maintenant. Loin s'origine dans trois dimensions principales, à la fois réelles et élaborées dans les imaginaires: l'espace du pays lointain, donnant à voir l'étendue que parcourt l'écrivain voyageur et son déplacement géographique, l'espace physique et symbolique des territoires, des chemins, des frontières et aussi l'espace du cosmos, de la recherche du même dans ce déplacement.

La dimension du temps - le temps biographique, le temps symbo- 
Un atelier d'écriture créative en FLE: tissages et apprentissages plurilingues

C. DOMPMARTIN-NORMAND,

A. Le Groignec lique - est travaillé dans le texte avec en particulier le rapport au temps dans la durée de l'écriture ou temps de l'invention.

La dimension de profondeur s'enracine dans la construction du sujet: elle participe de son histoire et de ses origines, de sa présence, de ses désirs. C'est la profondeur de l'informulé que l'écrivain tend à déchiffrer.

Dans ces trois dimensions se développe le rapport au monde spécifique à chacun-e et ce sont les vecteurs selon lesquels se développent aussi les habiletés de l'écrivain plurilingue vis à vis du langage en général et de ses fonctions.

Etre audible, compréhensible ici: à la lecture de leurs textes, grâce au retour organisé des commentaires suivant la lecture, les étudiants s'aperçoivent que plusieurs niveaux de perception sont sollicités par leurs textes.

D'une part, ils remarquent que les autres comprennent ou non, dans leur texte, tout ou partie des éléments qui permettent de saisir une situation, un personnage, un lieu...: de qui, de quoi s'agitil? quand? où? Leur écart expérientiel, leur diversité de provenance et de vécu augmentent la nécessité de la précision des références et de la contextualisation de leur texte. La fonction référentielle du langage est ainsi mise en lumière.

En même temps, ils font l'expérience qu'ils parviennent à faire passer une émotion, une vibration, une nuance, qu'ils ont voulu exprimer et que leur lecteur-auditeur signale avoir perçue, comme poésie particulière de leur texte. Cette expérience est particulièrement émouvante pour l'écrivain et la fonction expressive est puissamment illustrée. Pour donner à entendre au groupe, la lecture d'un texte peut être faite par un autre participant que son auteur. Dans l'une des séances, Mindaugas (Lituanie) lit son texte, mais sa prononciation est difficile à comprendre. Hamoud (Mauritanie) propose de lire le texte de Mindaugas. Il fait ce commentaire après avoir lu: "Les événements choisis sont magnifiques. Les idées viennent du profond. Il y a de la chaleur dans les mots» (Hamoud2013-ALG). Mindaugas écoute attentivement la lecture de son texte et montre son émotion. Ensemble, par l'écriture, l'écoute puis la réécriture, ils éprouvent la matière même de la langue autre dans sa fonction poétique.

Ils sont d'ailleurs souvent surpris que le texte produise à la lecture d'autres effets de sens que ceux qu'ils avaient voulu y mettre au départ, chez eux comme chez les autres.

"Quand Houria a lu mon texte, j'ai eu l'impression que c'était quelqu'un d'autre qui l'avait écrit, comme un écrivain (rires), j'ai trouvé plus beau dans sa bouche» 
Un atelier d'écriture créative en FLE: tissages et apprentissages plurilingues

C. DOMPMARTIN-NORMAND,

A. Le Groignec

\section{L'ÉCRIVAIN}

EN DEVENIR:

CONTINUITÉ

DE L'IDENTITÉ
(Kishidjagal. Mongolie-2014-CDN).

Et la prise de distance modifie la perspective:

«Ecrive c'est comme peindre, quand on a fini, on regarde le tableau et on s'aperçoit qu'ily a plus de perspective qu'on ne le pensait. Comme si le texte gagnait vie. Il trouve sa voie» (Anna-Luiza-Brésil-2013-ALG).

Quant à la dimension métalinguistique, elle surgit parfois spontanément dans leurs textes ou bien à l'occasion de propositions orientées dans cette direction.

«Une plume dans ma main, brouillard dans ma tête... D'où vient la levure pour mon texte?» (Corinna-Allemagne-2013-ALG).

Toutes ces dimensions font l'objet d'échanges. Ainsi, malgré les difficultés de prononciation, les erreurs morpho-syntaxiques, les approximations lexicales et de registre, leur texte est accueilli, écouté, ressenti, commenté. Malgré les entraves à l'expression qu'ils ressentent comme un manque, ils reçoivent des signes de reconnaissance du sens, ou plutôt des sens que les lecteurs découvrent et font connaître à l'écrivain dans leurs lectures plurielles.

Ainsi ils se reconnaissent dans le texte, le poème lu, entendu. Des complicités naissent, des rires, de l'émotion. Un soulagement à retrouver une communauté de langage dans cette autre langue, une speech community constituée de leur pluralité.

Dans cette expérience, ils éprouvent, semble-t-il, qu'une parole peut être «vraie», même si elle est formellement «incorrecte». «Vraie» dans le sens où l'entend Yves Bonnefoy déclarant:

«Le poème s'est avoué seulement de l'art, mais en cet aveu, il a fait acte de vérité, ce qui ouvre le champ d'une recherche du vrai où autrui s'implique, où une communauté se bâtit dans et par ce besoin de désassembler le vrai du faux, le tangible de l'illusoire» (Bonnefoy 2006).

L'atelier les place dans une activité d'écriture créative, artistique, que certains connaissent déjà dans d'autres langues. Mais pour la majorité, l'atelier inaugure une pratique poétique, inattendue, de la langue. Dans quelle mesure cette activité leur permet-elle de transporter dans leur texte, un sens profond, vivant, qui résiste aux chocs et aux ruptures de ce transport?

«fe n’aurais jamais pensé que j’aurais pu écrive ça en français» (Graciela-Bolivie2013-ALG).

«fe me déchiffre. Mon autre se sublime» (Anna-Luiza-Brésil-2013-ALG).

Ce qui est au cœur de leur activité est bien ce déchiffrage d'un informulé profond, essentiel et pas tant de «coller» par une traduction décevante à un énoncé qui serait déjà là dans leur langue. En cela il-elle est à la fois traducteur de sa langue première et traducteur de ce qu'il-elle n'a pas encore nommé. La traduction de cet infor- 
Un atelier d'écriture créative en FLE: tissages et apprentissages plurilingues

C. DOMPMARTIN-NORMAND,

A. Le Groignec
DU «MANQUE»

AUX «PLUS» INATTENDUS mulé est difficile, quelle que soit la langue, difficulté qu'expriment les étudiants lors des échanges sur les conditions de production de leurs textes. C'est le chemin d'émergence d'une parole vraie. Dans cet effort pour dire «ici» quelque chose du «loin», l'écrivain en langue autre s'aperçoit donc qu'il n'y a pas, d'une certaine manière, de différence de nature entre le travail d'écriture créative dans sa langue et en langue autre, mais qu'il peut, dans une continuité avec son être profond, poursuivre en français sa tentative de dire. D'où son soulagement, car l'enjeu est considérable, de continuer son existence dans cette autre langue.

«Pour moi ça ne fait pas de différence si je’erris en français ou en portugais» (AnnaLuiza-Brésil- 2013-ALG)

«C'est comme des musiciens qui parlent plusieurs langues: ils trouvent plus facile d'écrire une musique dans cette langue plutôt que dans telle autre») (Anna-LuizaBrésil-2013-ALG )

Par ailleurs, écrire matérialise une continuité: c'est une entreprise métaphorique, un transfert imaginaire qui permet de voyager en gardant le contact ici avec le lointain, sans se perdre. Les figures de style permettent d'opérer ce transfert en continuité, dans une métaphore au sens premier du terme (gr. metaphero/metaphora) c'est à dire un transport, un déplacement d'une langue à l'autre.

«Lorsque je veux écrire, mon cœur bat dans la cage thoracique de la langue» (Youcef-Algérie-2013-ALG).

«Ecrire pour moi est une expérience extravagante» dit Corinna (C.-Allemagne2013-ALG).

Extravagant, hors du chemin. Dans cette errance, il y a la peur de se perdre comme celle des voyageurs qui explorent les confins des territoires: la nuit, le brouillard des mots, les confins de la langue. Ce qu'ils-elles découvrent dans l'atelier, c'est qu'il est possible de ne pas se perdre en écrivant en français. Que le cœur continue à battre dans la langue, parce qu'ils parviennent à garder un contact avec eux-mêmes en écrivant. Ils se reconnaissent et ils sont reconnus, malgré le brouillard des mots qui noie des frontières vagues, incertaines, déplacées.

Comme mentionné plus haut, ils ont construit des représentations de l'apprentissage du français sur la base du manque, de l'imperfection.

A un premier niveau, l'atelier répond à leurs attentes de combler ce manque, et leur permet de progresser. En quelque sorte, s'opère une réparation narcissique dans l'atelier, par rapport aux autres cours où le manque de mots et le défaut de performance sont ressentis cruellement parfois. 
Un atelier d'écriture créative en FLE: tissages et apprentissages plurilingues

C. DOMPMARTIN-NORMAND,

A. Le Groignec
Là, d'entrée de jeu et au fil des semaines, ils découvrent que dans cette pénurie, ils accèdent parfois à un «plus» inattendu, car l'atelier leur permet de développer un faisceau d'habiletés reliées entre elles: celles de traducteur, d'expert de sa propre langue, d'expert de la langue autre, de lecteur critique de ses textes et de ceux des autres, enfin d'écrivain. Ce dernier point intègre et autorise les autres dimensions en ce qu'il permet l'augmentation du pouvoir d'agir (empowerment) et ces positions discursives jouent en circularité:

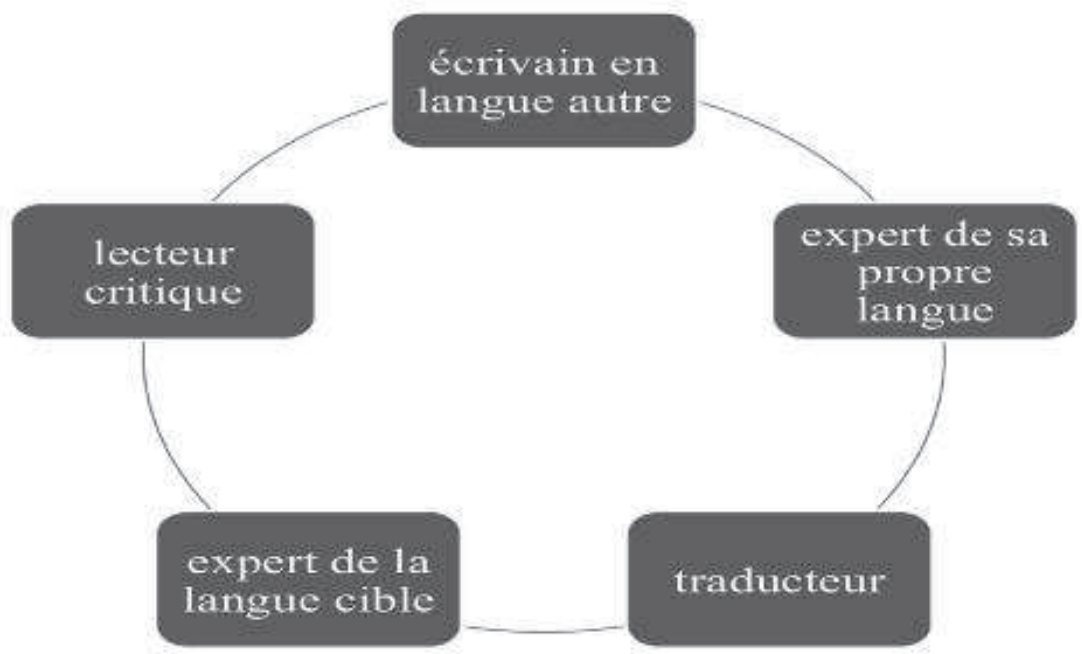

Dans l'atelier d'écriture, ils se découvrent écrivains, donc auteurs d'une parole singulière qui mérite d'être diffusée. Ils sont adoubés dans cette identité auctoriale par les autres.

«f'ai en face de moi une écrivaine. C'est magnifique ce que tu as écrit», dit Hamoud (H.-Mauritanie-2013-ALG) parlant à Anna-Luiza.

"C'est la première fois que j'écris des histoires en français» dit Graciela (G-Bolivie2013-ALG).

L'activité est vécue comme un accès à une liberté:

"C'est pas comme les autres cours, on est comme des écrivains. C'est génial, on laisse son âme libres (Kim-Vietnam-2013-ALG).

Elle est aussi un devenir: «fe cherche ma signature, elle n'est pas encore distincte» (Youcef-Algérie-2013-ALG).

«Je suis une écrivaine. Ca veut dire quoi? Ca ne parle pas des langues que j’utilise, mais des idées qui émergent dans cette langue. [...] Je suis une écrivaine et chaque langue que j'utilise est un autre conduit pour mes pensées [...]» (Suzanne-Australie-2013-CDN).

Par cette expérience, tout en gardant leur identité, ils cessent d'être des «étrangers» et rejoignent la communauté des écrivains. 
Un atelier d'écriture créative en FLE: tissages et apprentissages plurilingues

C. DOMPMARTIN-NORMAND,

A. Le Groignec

\section{BIBLIOGRAPHIE}

"L'échange interculturel m'a aussi beaucoup fait penser. Maintenant (peut-être) je comprends mieux l'universalité des écrivains». (Andreea-Roumanie-2013-ALG)

Dans l'atelier et le travail sur l'expression par le biais de l'écrit est remis en lumière le véritable enjeu de la compétence langagière, à envisager comme globale, holistique, pluri-littératiée et pluristyle. Pour tout un chacun, acteur social composite, (mé)tissant ses expériences au fil de ses déplacements et de sa vie, il s'agit de l'accès à la parole, laquelle préfère quelquefois, dans un apparent paradoxe, les chemins de traverse:

«Je ne cesse finalement de me rendre étranger à moi-même dans les deux langues $[. .$.$] toujours décalé, hors de place, à coté de tout ce qu'exige de$ moi la liturgie sociale de l'une et de l'autre langue. Mais c'est justement de ce lieu écarté que j’accède à la parole» (Mizubayashi 2011: 267).

BILLIEZ J. (ed.). 1998. De la didactique des langues à la didactique du plurilinguisme. Hommage à Louise Dabène, Grenoble: CDL-LIDILEM. BONIFACE C. 1992. Les ateliers d'écriture. Paris: Retz.

BONNEFOY Y. 2006. La poésie comme acte de vérité. Le Monde, 30 juin 2006.

CANAGARAJAH S. 2011. Codemeshing in Academic Writing: Identifying Teachable Strategies of Translanguaging. In Toward a Multilingual Approach in the Study of Multilingualism in School Contexts, The Modern language journal, 95-3: 401-417.

CUMMINS J. 2013. Current research on language transfer: Implications for language teaching policy and practice, pp. 289304 in P. SIEMUND et al. (ed.), Multilingualism and language diversity in urban areas: Acquisition, identities, space, education. Frankfurt: Peter Lang.

DOMPMARTIN G., GALLIGANI S., HONJO T. \& AUDOGIANOTTI S. 2013. Quand le français, l'anglais et le japonais se mêlent dans les échanges familiaux: un tricotissage créatif des ressources plurilingues, pp. 97-106 in V. BIGOT, A. BRETEGNIER \& M.-T. VASSEUR, Vers le plurilinguisme? Vingt ans après, Paris: Editions achives contemporaines.

GLISSANT E. 1993. Entretien avec Lise Gauvin. Littératures $n^{\circ}$ 12, Strasbourg: Édition spéciale du Carrefour des littératures européennes.

HAMERS J. \& BLANG M. 1983. Bilingualité et Bilinguisme, Bruxelles: Pierre Mardaga.

HUSTON N. 2005. Longings and belongings, Essays. Toronto: McArthur and Company.

HUSTON N. 1999. Nord Perdu. Paris: Actes Sud.

KRISTEVAJ. 1988. Etrangers à nous-mêmes. Paris: Fayard. 
Un atelier d'écriture créative en FLE: tissages et apprentissages plurilingues

C. DOMPMARTIN-NORMAND,

A. Le Groignec
MIZUBAYASHI A. 2011. Une langue venue d'ailleurs, Paris: Gallimard.

PERREGAUX C. 2004. Prendre appui sur la diversité linguistique et culturelle pour développer aussi la langue commune. Repères $\mathrm{N}^{\circ} 29$.

PRASAD G. 2012. Multiple Minorities or Culturally and Linguistically Diverse (CLD) Plurilingual Learners? Re-envisioning Allophone Immigrant Children and their Inclusion in French-Language Schools in Ontario. The Canadian Modern Language Review/La Revue canadienne des langues vivantes, 68-2: 190215.

SAYAD A. 1999. La double absence. Des illusions de l'émigré aux souffrances de l'immigré. Paris: Seuil.

\section{NOTES}

(1) Cette compétence plurielle concerne à notre sens tout sujet parlant, si l'on inclut dans sa définition la dimension pluristyle, à savoir la capacité à utiliser des styles (et registres sociaux) variés. Ceux-ci sont considérés comme des variations dans un seul «code», mais témoignent néanmoins d’habiletés à «switcher», souvent méconnues ou inconscientes.

(2) «l'interférence désigne des problèmes d'apprentissage dans lesquels l'apprenant transfêre le plus souvent inconsciemment et de façon inappropriée des éléments et des traits d'une langue connue dans la langue cible» (Hamers et Blanc 1983: 452).

(3) "The I which I used so freely in my essays was also, no doubt, one of the effects of my uprooted knowledge. A certain shamelessness was made possible by the fact I was writing in a foreign language partly because, at least in my imagination, my parents did not speak this language, but more importantly because for me, French has nothing to do with my intimate, inner life. In French I could say, quite calmly and even with a certain indifference, things it could have been impossible for me to reveal or even think about in my mother tongue" (Huston 2005: 341).

(4) Conventions de transcription du corpus: en italiques, les propos d'étudiants recueillis sur le carnet d'ethnologue de l'animatrice; entre parenthèses: prénom de l'étudiant-e, pays de provenance, année recueil, ALG= corpus Anne Le Groignec, CDN= corpus Chantal Dompmartin. Toutes les citations d'étudiant.e.s sont issues du corpus des ateliers 2013-2014. Certain.e.s étudiant.e.s ont souhaité que seul leur prénom soit cité: Anna-Luiza, Graciela, Kim, Mindaugas, Naoko, Youcef, Houria, Kishidjargal, Suzanne; d'autres que leur nom soit également indiqué: il s'agit de Corinna Arnold-Smaus, Andreea Bebec-Krause, Hamoud Blal, Natalia Haber. Qu’ils/elles soient tou.te.s remercié.e.s pour nous avoir autorisées à citer leurs textes et propos. 